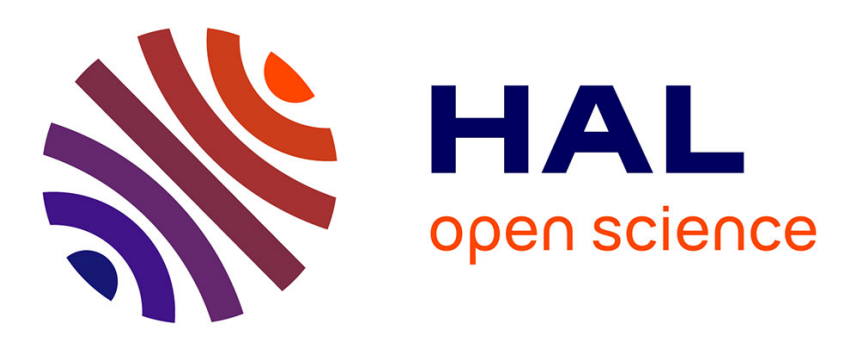

\title{
Du théâtrophone au théâtre pour extensions mobiles : présences à distance dans les téléscènes
}

Clarisse Bardiot

\section{To cite this version:}

Clarisse Bardiot. Du théâtrophone au théâtre pour extensions mobiles : présences à distance dans les téléscènes. Josette Féral. Pratiques performatives: body remix, Presses universitaires de Rennes, pp.315-332, 2012, 10.4000/books.pur.80831 . hal-02337817

\section{HAL Id: hal-02337817 https://hal.science/hal-02337817}

Submitted on 29 Oct 2019

HAL is a multi-disciplinary open access archive for the deposit and dissemination of scientific research documents, whether they are published or not. The documents may come from teaching and research institutions in France or abroad, or from public or private research centers.
L'archive ouverte pluridisciplinaire HAL, est destinée au dépôt et à la diffusion de documents scientifiques de niveau recherche, publiés ou non, émanant des établissements d'enseignement et de recherche français ou étrangers, des laboratoires publics ou privés. 


\section{DU THÉÂTROPHONE AU THÉÂTRE POUR EXTENSIONS MOBILES : PRÉSENCES À DISTANCE DANS LES TÉLÉSCÈNES}

\section{Clarisse Bardiot}

« Si nous accordons la conscience, et tout ce qui nous distingue des objets, aux personnes qui nous entourent, aucun argument valable et sans réplique ne nous permettra de la refuser aux personnes créées par mon appareil. »

Adolfo Bioy Casares, L'Invention de Morel, 1940

De plus en plus d'artistes déclarent « faire du théâtre » pour des dispositifs de présence à distance, qu'il s'agisse du réseau ou de nos extensions électroniques mobiles (téléphones portables, PDA, Ipod...). On peut regrouper ces différentes pratiques sous le terme générique de téléscène. Les téléscènes sont des scènes en réseau. Ces « scènes à distance " peuvent être soit des lieux numériques, comme des forums ou des environnements virtuels disponibles sur Internet, soit des lieux physiques, comme des plateaux de théâtre ou des cybercafés reliés par Internet, ou par un réseau créé pour l'occasion. Les combinaisons de lieux numériques, hébergés sur des serveurs, avec des lieux physiques, sont multiples. La scène et la salle ne sont plus des entités circonscrites, mais éclatées, démultipliées, dispersées, leur nature et leur taille variant au rythme des connexions.

Parmi la multiplicité des formes qu'a pu prendre le théâtre, un seul paramètre ne varie pas : la co-présence physique en un même lieu et un même temps des acteurs et des spectateurs. Cette co-présence est caractérisée par une absence de médiation (c'est ainsi que Benjamin distingue le théâtre du cinéma ${ }^{1}$ ). Or l'une des caractéristiques des téléscènes est justement l'absence de co-présence dans le hic et nunc de la représentation, avec pour corollaire la médiation : les spectateurs et les acteurs se rencontrent et communiquent entre eux par le réseau, par le biais d'interfaces numériques. La médiation serait synonyme d'une perte de présence : les acteurs seraient absents aux spectateurs, et chaque spectateur serait absent aux autres spectateurs - ce qui fait ressurgir la notion de public ${ }^{2}$. Dans les téléscènes,

${ }^{1}$ Benjamin W., «L'Euvre d'art à l'époque de sa reproductibilité technique » (1939), in CEuvres III, Gallimard (coll. Folio essais), Paris, 2000, pp. 269-316.

${ }^{2}$ Pour Mario Costa, qui a défini avec Fred Forest «l'esthétique de la communication », «la question du «public » et de sa re-définition est un aspect fondamental du travail esthétique lié à la communication à 
où est le public et comment sa présence se manifeste-t-elle ? Faut-il représenter le public et créer une salle virtuelle ? Ou au contraire faut-il l'abstraire de la représentation et lui laisser simplement la possibilité de se connecter ? Dans quelle mesure peut-il réagir ou participer ? Dispersés aux quatre coins du monde, acteurs et spectateurs sont confrontés à un « drame des distances $\gg{ }^{3}$ qui permet de repenser aujourd'hui l'organisation spatiale de la scène et de la salle, de l'espace de l'action et de l'espace de l'écoute et du regard.

\section{Premières expériences}

Si la téléprésence semble un phénomène récent au théâtre et lié à l'explosion d'Internet, on peut en trouver les prémices dans les trois grands procédés de présence à distance qui se développent au $\mathrm{XX}^{\mathrm{è}}$ siècle : téléphone, radio et image électronique.

\section{Le théâtrophone}

L'une des toutes premières applications du téléphone est le théâtrophone, une invention mise au point par Clément Ader pour l'Exposition internationale d'électricité à Paris en 1881. Le public pouvait entendre en direct, et individuellement, grâce à deux écouteurs, les spectacles joués à l'Opéra, situé à plus de deux kilomètres. Par la suite, la « compagnie du théâtrophone " propose à ses abonnés des spectacles joués dans divers théâtres parisiens. Le théâtrophone transforme l'expérience publique du théâtre en expérience privée et permet de multiplier les types de publics : on peut d'ores et déjà parler de salle partagée, avec des spectateurs présents dans la salle traditionnelle, et de salle distribuée, avec des spectateurs éparpillés à chacun des bouts du réseau téléphonique relié au théâtre. De plus, la place des « transmetteurs», disposés entre les chanteurs et l'orchestre, transporte le spectateur connecté à distance au centre de la scène, et non devant la scène, à un lieu d'écoute inédit et privilégié qui favorise un sentiment d'immersion.

\section{La radio}

«La radia sera [...] luttes des bruits et des distances différentes, c'est-à-dire le drame spatial ajouté au drame temporel ». Marinetti et Masnata, La Radia, $1933^{4}$

En octobre 1933 Marinetti et Masnata publient un manifeste intitulé La Radia dans la Gazzetta del Popolo. La Radia est le nom donné « aux grandes manifestations de la radio ». Considéré comme annonciateur de la réflexion contemporaine sur les réseaux et des propositions artistiques liées aux technologies de l'information et de la communication, ce

distance : d'une fois sur l'autre, elle le détruit, l'intègre, l'exclut, le resserre, l'élargit indéfiniment... », voir Costa M., Internet et globalisation esthétique. L'avenir de l'art et de la philosophie à l'époque des réseaux. Traduit par Giordano Di Nicola, Paris : L’Harmattan, coll. « Ouverture philosophique », 2003, p. 70.

${ }^{3}$ Titre de l'une des Cinque Sintesi dal Teatro Radiofoniche écrites par Marinetti en 1933.

${ }^{4}$ Nous utilisons tout au long de cet article la traduction publiée in Bureaud A. et Magnan N. (dir.), Connexions. Art, réseaux, médias, École Nationale Supérieure des Beaux-Arts, Paris, 2002, p. 270-273. 
manifeste est très souvent mentionné dans les études sur l'histoire des nouveaux médias, de la téléprésence ou encore au sujet du net $\operatorname{art}^{5}$.

Au moment où l'usage du téléphone et de la radio se répand, les futuristes prennent la pleine mesure du bouleversement induit par la téléprésence. Marinetti expérimente le don d'ubiquité du téléphone dans la soirée futuriste du 17 mai 1914, à Rome. Alors à Londres, il intervient dans cette soirée par téléphone interposé, réalisant ainsi ce qui est sans doute la première performance à distance de l'histoire du théâtre ${ }^{6}$. Quelque temps avant la publication de $L a$ Radia, plusieurs textes et manifestes appellent à l'intégration de la radio et de la télévision au théâtre (les premiers tests en Italie sur la télévision ont lieu au début des années 1930) : en avril 1931, Marinetti publie « Manifesto del Teatro Aereo radiotelevisivo ${ }^{7}$ »; dans Teatro totale per le masse (Théâtre total pour les masses), en janvier 1933, il appelle de ses vœux un théâtre total qui réunirait « le cinéma - la radio - le téléphone - la lumière électrique - le néon - l'aéropeinture - l'aéropoésie - le tactilisme - l'humour et le parfum ", et aurait recours à des écrans de télévision ${ }^{8}$; dans le Manifeste sur le théâtre futuriste (avril 1933), Fernando Raimondi souhaite que « cinématographe, radio et télévision [soient] au service du théâtre futuriste ${ }^{9} \gg$.

La Radia marque une rupture avec les textes antérieurs : si les futuristes voyaient dans les nouvelles technologies de leur époque (radio, télévision, cinéma) un moyen efficace de renouveler le théâtre, cet optimisme semble définitivement mis au rebus. La radia signe la mort du théâtre :

« La radia ne doit pas être

Théâtre, par ce que la radio a tué le théâtre, déjà vaincu par le cinéma sonore ».

Marinetti et Masnata insistent, s'en prenant radicalement à toutes les caractéristiques du théâtre classique, dans une mise en pièces sans concession :

«La radia abolit

L'espace nécessaire dans le théâtre, y compris le théâtre synthétique futuriste (action sur une scène fixe et constante) et dans le cinéma (actions très rapides et très variables, simultanées et toujours réalistes).

Le temps

L'unité d'action

Le personnage théâtral

Le public, en tant que juge autoélu, hostile et toujours rétrograde ».

\footnotetext{
${ }^{5}$ Par exemple : « La Radia predicts the shift from industrial world to postindustrial world, from a machine age to an electronic age, moving beyond radio into the kind of global information that has since come to be », voir Lander D., «Radiocasting : Musings on Radio and Art », Augaitis D. et Lander D. (dir.), Radio Rethink : Art, Sound and Transmission, Banff, juin 1994, p. 16.

${ }^{6}$ Lista G., « Art et technologie », in Ligeia, $\mathrm{n}^{\circ}$ 45-48, juillet-décembre 2003, p. 6.

${ }^{7}$ Lista G., Théâtre futuriste, T. II, L'Age d'Homme, Lausanne, 1976, p. 247.

${ }^{8}$ Lista G., Futurisme. Manifestes, documents, proclamations, L'Âge d'Homme, Lausanne, 1973, p. 285.

${ }^{9}$ Lista G., Théâtre futuriste, op. cit., p. 212.
} 
La Radia se veut une entreprise de destruction non seulement des trois unités de temps, de lieu et d'action, mais aussi du personnage et du public. Si le théâtre est ainsi déclaré art périmé, c'est notamment parce que la scène "non plus visible, ni encadrable » devient « universelle et cosmique ». La radio permet à l'auditeur immobile de franchir continents et fuseaux horaires, dans un télé-scopage de lieux et de temps hétérogènes. L'unité d'action est alors remplacée par des « actions simultanées ». Du personnage théâtral, il ne reste plus que la voix et la parole, thème qui sera développé dans des manifestes futuristes ultérieurs. Enfin le public, éclaté en multiples individus éparpillés sur la planète, ne saurait être considéré comme tel.

Toujours en 1933, Marinetti rédige Cinque Sintesi dal Teatro Radiofoniche, dont Le Drame des distances. Ces synthèses ne seront pas réalisées à cause de l'insuffisance des techniques de l'époque.

Par ailleurs, dès les années 1920, le théâtre radiophonique connaît un véritable succès et des auteurs de théâtre écrivent des pièces spécifiquement pour ce média. Ainsi le jeune Brecht conçoit pour la radio Vol au-dessus de l'océan en 1927. Pourquoi peut-on encore parler de théâtre ? D'une part les pièces radiophoniques suscitent des formes d'écoutes collectives. D'autre part, lorsque la radio relève d'une expérience privée, Mc Luhan remarque que l'écoute de la radio a malgré tout le pouvoir «de transformer presque instantanément l'individualisme en collectivisme ${ }^{10}$ ", « l'individu et la société en une seule et même chambre réverbérante ${ }^{11}$ ». Avec ce « théâtre pour l'oreille », l'auditeur visualise la représentation, il la complète avec des images mentales, alors qu'avec le téléphone, « l'image acoustique [étant] extrêmement pauvre, nous la renforçons et la complétons au moyen de tous nos autres sens ${ }^{12} »$. Cette différence de qualité de l'image acoustique expliquerait en partie pourquoi un théâtre radiophonique s'est développé, et non un « théâtre téléphonique ».

Dans la lignée de ses expériences radiophoniques, Brecht propose un théâtre à distance qui permette la réaction du spectateur: «Il faut [...] transformer [la radio] d'appareil de distribution en appareil de communication. La radio pourrait être le plus formidable appareil de communication qu'on puisse imaginer pour la vie publique, un énorme système de canalisation, où plutôt elle pourrait l'être si elle savait non seulement émettre, mais recevoir, non seulement faire écouter l'auditeur, mais le faire parler, ne pas l'isoler, mais le mettre en relation avec les autres. $\rangle^{13}$

\section{L'image électronique}

Une autre étape décisive vers les téléscènes est la mise au point de l'image électronique, laquelle réalise le vieux rêve de la vision à distance et donne naissance à la télévision. Audelà des diverses expériences de diffusion de pièces de théâtre pour la télévision, l'image

${ }^{10}$ Mc Luhan M., Pour Comprendre les médias, Paris : Le Seuil, 1968, p. 346.

${ }^{11}$ Id., p. 342.

${ }^{12}$ Idem, p. 308.

${ }^{13}$ Brecht B., « Théorie de la radio » (1927-1932), in Ecrits sur la littérature et l'art 1, Paris : L'Arche, 1970, p. 137. 
électronique s'invite sur le plateau, provoquant l'intrusion en direct d'événements qui ont lieu en-dehors de la scène. Ainsi, le scénographe tchèque Svoboda a recours pour la première fois en $1965^{14}$ à un réseau de télévision en circuit fermé pour un opéra, Intolleranza, de Luigi $\mathrm{Nono}^{15}$. Ce dispositif permet à Svoboda de capter et de diffuser en direct différentes images, notamment une manifestation contre le spectacle qui a lieu en même temps à l'extérieur du théâtre, des actions dans les coulisses ou encore le public.

A la recherche de nouveaux moyens d'expression, Jacques Polieri a mené à partir des années 1950 une réflexion sur l'usage de l'image électronique au théâtre. Pour lui, l'image électronique est « un nouveau type de relais entre la scène et l'œil du spectateur; en ce sens elle va peut-être conduire à une nouvelle forme de scénographie, à un nouveau genre de spectacle ${ }^{16}$ ». Poursuivant cette réflexion, Polieri définit en 1963 une "scénographie de l'image électronique ${ }^{17} \gg$ dont les principes sont : projections électroniques sur des écrans géants, instantanéité, possibilité de voir à distance, placement du spectateur à la place du réalisateur en régie en lui donnant plusieurs vues simultanées, modification de la forme des écrans. Polieri applique ces réflexions dans différents " jeux de communication », dont le premier est celui réalisé en 1972 pour la Rue des loisirs des Jeux Olympiques de Munich. La mise en réseau des différentes sources, ainsi que le recours au direct, permet aux spectateurs de communiquer avec d'autres spectateurs à distance, via des images et du son. Ce système détruit le mode de communication pyramidale de la télévision et fait apparaitre une nouvelle figure, celle d'un meneur du jeu, à la fois modérateur et initiateur des actions à distance.

Dans les projets de Kitt Galloway et Sherrie Rabinowitz, lesquels souhaitent s'écarter des espaces de représentation traditionnels ${ }^{18}$, l'image électronique devient le lieu de la rencontre. Il ne s'agit plus tant de relier des spectateurs que de faire partager un même espace de jeu à des acteurs présents dans différents lieux, de créer un « espace image distribué $»^{19}$. En 1977, dans Satellite Arts Project, deux danseurs séparés par quatre mille kilomètres sont réunis dans une image électronique, grâce à une liaison satellite et le concours de la NASA. Chaque danseur est filmé, et les deux sources sont mélangées afin de créer une seule image, véritable lieu du spectacle. Kitt Galloway et Sherrie Rabinowitz proposent ainsi une scène sans limite géographique, se libérant des contraintes de l'espace physique. A la même période, et dans le même ordre d'idées, Myron Krueger imagine un "théâtre distribué »" à la suite de son installation Videoplace.

\footnotetext{
${ }^{14}$ Il s'agit de la version présentée à Boston. Une première version a été présentée à Venise en 1961, mais les images initialement prévues ont été remplacées par des projections abstraites.

${ }^{15}$ Bablet D., Josef Svoboda, Paris : La Cité, 1970 ; réédition revue et complétée : L'Age d'Homme, coll. th XX, Lausanne : 2004, p. 135.

${ }^{16}$ Polieri J. Scénographie nouvelle, Architecture d'Aujourd'hui, 1963 ; réédition revue, corrigée et augmentée sous le titre Scénographie. Théâtre, cinéma, télévision, Paris : Jean-Michel Place, 1990, p. 109.

${ }^{17}$ Polieri J., Oudin M., « Scénographie de l'image électronique », Polieri Jacques, Scénographie, op. cit., p. 129.

${ }^{18}$ Kit Galloway à Clarisse Bardiot, mail du 27 juin 2003.

${ }^{19} \mathrm{Idem}$.

${ }^{20}$ Krueger M. W., Artificial Reality II, Addison-Wesley, 1990, p. 221-222.
} 
Du théâtrophone à l'image électronique, la présence à distance implique toujours davantage le spectateur. Ce n'est plus seulement l'acteur qui se déplace au domicile de chacun, mais le spectateur qui se trouve aussi affecté d'une mobilité dans les réseaux. Le spectateur n'est plus seulement un récepteur mais à la fois émetteur et récepteur. Internet va démultiplier ce processus. Avec l'informatique jointe au réseau - la " télématique $»^{21}-$ non seulement le spectateur est doué d'ubiquité, mais de plus cette ubiquité prend un nouveau sens : il ne s'agit plus seulement du déplacement d'une image et d'une voix, de la vue et de l'ouie, mais du corps : il est ainsi possible de diriger des robots à distance, ou encore de toucher des objets à des milliers de kilomètres, grâce à des systèmes à retour d'effort.

\section{Délocaliser dans le cyberespace}

« Je ne sais si jamais philosophe a rêvé d'une société pour la distribution de Réalité Sensible à domicile ». Paul Valéry, La Conquête de l'ubiquité, $1928^{22}$

Plusieurs metteurs en scène, souvent issus du monde universitaire, s'emparent dès leur création des espaces partagés sur le réseau, comme les MUD, MOO et autres espaces de chat, puis des environnements en VRML, et enfin aujourd'hui de Second Life, pour y jouer des pièces de théâtre. Définir des stratégies du regard, organiser le point de vue : tel est l'enjeu de ces représentations sur Internet. En effet, le changement majeur par rapport à une mise en scène sur un plateau consiste en la dispersion de la troupe et du public dans le monde entier. Connectés entre eux par Internet, acteurs et spectateurs ne partagent pas un même espace physique. Seul l'espace numérique permet de les réunir.

Après quelques expérimentations en 1992, Stuart Harris crée en 1993 les Hamnet Players, qu'il présente comme une compagnie jouant « des parodies de Shakespeare et de Tennessee Williams dans des environnements IRC $»^{23}$. En décembre 1993, ce groupe monte la première pièce jouée dans un chat, Hamnet, une adaptation de Hamlet. La représentation commence ainsi :

« $<$ PROLOGUE $>$ All the world's a Unix term... [3]

$<$ PROLOGUE $>\ldots$ and all the men \& women merely irc addicts... [4] $\gg^{24}$

Dans ce type de performance, c'est l'écriture elle-même qui devient spectacle : la représentation consiste à improviser sur une trame définie en envoyant dans un chatroom des messages textuels. Dès les premières expériences sont posées les questions de l'adaptation

\footnotetext{
${ }^{21}$ Terme inventé par Simon Nora et Alain Minc à partir de télécommunication et informatique, à l'occasion d'un rapport remis au président Valery Giscard D'Estaing,; voir Nora S., Minc A., L'Informatisation de la société, Paris : La Documentation française, 1978.

${ }^{22}$ Valéry P., « La Conquête de l'ubiquité » (1928), in CEuvres II,Paris, NRF, Bibliothèque de la Pléiade, 1960, p. 1285.

${ }^{23}$ Pfeiffer, G. « Theatrical Performance in the World Wide Web », in Trans, ${ }^{\circ} 9$, décembre 2000. Disponible en ligne à [http://www.inst.at/trans/9Nr/pfeiffer9.htm]. IRC (Internet Relay Chat) est un protocole de chat.

${ }^{24}$ Script de Hamnet, The Hamnet Players, décembre 1993, [http://www.hambule.co.uk/hamnet/hscript.htm]
} 
d'un texte existant, des modes de représentation du jeu des acteurs, de la présence et du rôle du spectateur. Ainsi, dans Hamnet, les signes de ponctuation sont utilisés pour dessiner les décors ou donner des indications sur le jeu. De plus, pour aider le spectateur à imaginer le jeu des acteurs, des personnages sont inventés, comme Percussions ( Drum»), Couleurs («Colors »), Action («Action »), et Sortie («Exit»). Développés dans les années 1980, les $\mathrm{MOO}^{25}$ fonctionnent sur le même principe. Antoinette Lafarge avec sa compagnie, les Plaintext Players, a commencé à explorer les représentations théâtrales dans les MOO en 1994. Elle a été suivie par les membres de la communauté virtuelle ATHEMOO en 1995.

L'invention du chat visuel en 1994, dont le Palace est l'exemple le plus célèbre, offre des possibilités qui vont au-delà des simples échanges textuels des débuts. Gratuit, ne nécessitant qu'une faible connexion téléphonique et simple à utiliser, le Palace est une application qui a suscité un engouement sans précédent. Des lieux virtuels en 2D figurent une pièce, un paysage, ou encore un espace abstrait. Chaque personne connectée y est représentée par un avatar de petites dimensions qu'elle conçoit elle-même de toutes pièces ou à partir des outils qui sont livrés avec l'application. Une fenêtre en bas de l'écran lui permet d'écrire un texte qui s'affiche aussitôt dans une bulle au-dessus de son avatar. Le texte peut être prononcé par une voix électronique grâce à des plug-in spécifiques. De façon générale, l'esthétique s'inspire de la bande dessinée, en particulier par la présence des phylactères, le type de graphisme et les modes de circulation du regard dans l'image.

À partir de 1996, des expériences de représentation théâtrale dans les chats visuels apparaissent. À ma connaissance, la première est celle de la Virtual Drama Society au Crosswaves Festival en mars 1996 à St Walnut aux USA. En 1997, Lisa Brenneis, réalisatrice de produits multimédias interactifs, et Adriene Jenik, artiste multimédia et enseignante d'art numérique à l'Université de Californie, fondent Desktop Theater. Ce terme désigne à la fois le nom de la compagnie et un nouveau genre de spectacle dramatique qui a lieu sur les écrans de nos ordinateurs, dans les salons des chats visuels du Palace.

Le premier spectacle de Desktop Theater conçu pour ce théâtre de rue en ligne ( «Internet street theater $»^{26}$ ) est Waitingforgodot.com, une adaptation de la pièce de Beckett. Au cours de la représentation, les chateurs interrogent les personnages, aident « Didi » et « Gogo » à retrouver Godot sur le serveur, et manifestent parfois le désir de connaître la fin de l'histoire, ou encore de mettre un terme à la non-action de Waitingforgodot.com. Ainsi, un chateur baptisé Muscleman a changé son nom en Godot au cours d'une représentation ${ }^{27}$. Par la suite, Desktop Theater a souhaité développer la partie improvisée de ses spectacles, en exploitant et en intégrant au mieux les interruptions des chateurs. Dans le sillage de Desktop Theater,

\footnotetext{
${ }^{25}$. Un MOO est un MUD (Multi-User Dimensions ou Dungeons) dont la programmation est orientée objet.

${ }^{26}$ Jenik A., « Desktop Theater, Keyboard Catharsis and the Masking of Roundheads », in The Drama Review, 45, 3 (T 171), MIT Press, automne 2001, p. 100.

${ }_{27}$ Evénement rapporté in Scott Rosenberg, Clicking for Godot, 2 octobre 1997, [http://www.salon.com/21st/feature/1997/10/02godot.html].
} 
d'autres compagnies comme [abc]experiments et Avatar Body Collision créent des spectacles pour des chats visuels.

L'apparition des webcams permet d'autres jeux, qui engagent l'image, et parfois la voix, du spectateur. Ainsi, Agnès de Cayeux, dans ses différentes créations pour le réseau, s'inspire des chambres de discussion video-chat du réseau Internet. Dans sa dernière création, In My Room (2005), une installation, un site Internet et un livre se répondent, offrant trois fenêtres différentes sur l'expérience de la rencontre et du désir sur Internet :

«In my room désigne ces singulières relations à distance - ces nouvelles interactions sensorielles et intellectuelles. Il s'agit d'interroger le réseau Internet, des usages que nous en avons à la « nature » que nous pouvons en pressentir. Le corps comme espace public, ce retrait radical de l'intime. Il s'agit aussi de rêver à une définition du réseau qui s'éloignerait radicalement d'un tout marchand et pornographique. L'utopie désignée. Ce réseau-là comme une préfiguration intelligente et sensible de notre propre évolution ${ }^{28} \gg$.

Dans les chats, il est possible de créer plusieurs espaces et d'attribuer un degré d'action plus ou moins grand à chaque personne en fonction de son rôle (spectateur, acteur, metteur en scène...) et du lieu virtuel auquel il se connecte. Il est ainsi possible d'interdire de parler aux spectateurs et de les laisser s'exprimer seulement par réactions prédéfinies (ennui, rires, soupirs...). Dans les représentations des Hamnet Players, un personnage, "Audience », représente les actions supposées du public connecté. Cependant, ce personnage ne se substitue pas aux membres du public, qui peuvent tous s'exprimer et intervenir dans la représentation, le plus souvent sous la forme de commentaires à chaud sur l'action. Dans les représentations de Desktop Theater, acteurs et spectateurs ne sont pas séparés, à tel point qu'il est parfois difficile de les distinguer les uns des autres ou que les chateurs ne se rendent pas compte qu'ils se trouvent au beau milieu d'une représentation théâtrale. S'inspirant des théories d'Augusto Boal, Desktop Theater joue de cette possible confusion.

De même que pour les chats, quasiment dès leur invention, différents types d'environnements virtuels partagés en 3D donnent lieu à des représentations théâtrales. Ainsi, pour Stephan Matsuba et Bernie Roehl, qui ont contribué à la création du VRML, « le monde entier est sans doute une scène, mais les mondes virtuels sont encore davantage : ils n'ont pas de quatrième mur, ni de coulisses ; ce sont des théâtres de l'esprit, dans lesquels nous jouons tous notre rôle, et où en plus nous l'écrivons $»^{29}$. En avril 1998, ils créent une adaptation du Songe d'une nuit d'été, intitulée VRML Dream, pour un environnement conçu en VRML. Chaque spectateur peut sélectionner des points de vue prédéterminés, suivre celui du metteur en scène (point de vue par défaut), regarder le spectacle à travers les yeux de l'un des personnages ou se promener librement dans le décor. Il n'est donc pas représenté, et n'a aucune notion du nombre, de la réaction ou de la position des autres spectateurs. Tout se

\footnotetext{
${ }^{28}$ Agnès de Cayeux, dossier de presse de In My Room, 2005.

${ }^{29}$ Matsuba S. N., Roehl B., " Bottom, Thou Art Translated: The Making of VRML Dream », in Computer Graphics Applications, vol. 19, n² 2, mars-avril 1999, p. 45.
} 
passe comme si la représentation n'avait lieu que pour lui seul. Cependant, il ne peut interagir avec les personnages. Sa seule liberté, c'est celle du mouvement, mais elle est sans conséquence sur la représentation. D'autres environnements créés avec des logiciels similaires voient fleurir des représentations théâtrales : Huit Clos de Sartre est adapté en 1998 dans un espace partagé avec le logiciel DIVE (Distributed Interactive Virtual Environment), développé par le Swedish Institute of Computer Science (SICS) ; Avatar Farm, projet mené par l'université de Nottingham en juin 2000, se déroule dans son propre système d'environnement virtuel partagé, Massive-3 ; Eva et Franco Mates recréent des performances sur Second Life.

La compagnie britannico-catalane Dogonefff, composée de Maria de Marias et d'Andrew Colqhuoun, s'attache à «penser le théâtre dans le contexte d'Internet ${ }^{30}$ ». La notion de « corps sans organes » d'Artaud, revue par Deleuze, est à la base de leurs recherches dont une étape importante est le cycle Teatro virtual créé en 2001. Les quatre premiers actes de Teatro virtual sont des représentations uniquement sur Internet. Elles consistent en la diffusion en flux continu et en direct (« livestream ») d'images vidéo sur Internet. On y voit Maria de Marias procéder à diverses performances rituelles, à des gestes répétitifs. Le cinquième acte de Teatro Virtual est une représentation sur un plateau qui convoque des images empruntées à Internet (chat, webcam, sites Internet...) diffusées en direct sur un écran placé en fond de scène. Dogonefff attache une importance particulière à la diffusion en direct des images. Pourtant il ne s'agit absolument pas d'une retransmission à distance d'un spectacle qui se déroulerait dans un lieu éloigné. Le livestream permet au contraire de communiquer au spectateur une intensité, une énergie convertie en durée. Le corps sans organes devient pure énergie grâce au flux du réseau.

Capter le flux du réseau et le rendre visible ou plutôt sensible : il semblerait qu'au-delà des sujets traités et des postures, les spectacles qui font intervenir des scènes connectées à distance ne visent qu'à cela. Dans les "sautes » d'images dues au rafraîchissement des données de la webcam de la fin de La Génisse et le Pythagoricien, conçu et mis en scène par Jean-François Peyret au TNS en 2002, ou bien dans la tentative de créer un personnage à partir de plusieurs acteurs filmés à distance dans les spectacles du Gertrud Stein Repertory Theatre, le spectateur est témoin des saccades du temps et de la collusion d'espaces éloignés fondus dans une durée commune.

Dans ces représentations, qu'elles se déroulent dans des chats, dans des environnements virtuels ou dans différents lieux reliés entre eux par un réseau, plusieurs cas de figures se présentent, essentiellement liés au désir d'impliquer ou non le spectateur dans la représentation. On peut distinguer trois stratégies différentes : le spectateur invisible, le spectateur discret et le spectateur invité. Le spectateur invisible a une liberté de mouvement, mais sa présence n'est pas manifeste aux yeux des acteurs et des autres spectateurs. Le spectateur discret est représenté (sous forme d'un chiffre indiquant le nombre de personnes

\footnotetext{
${ }^{30}$ Propos recueillis à Barcelone en octobre 2003.
} 
connectées, par un pseudonyme qui s'affiche à l'écran ou par un avatar) mais il ne peut réagir que de façon limitée sur la représentation, voire pas du tout, et sa situation est très proche du spectateur invisible. Enfin, le spectateur invité « monte » sur scène. Il est représenté, le plus souvent par un avatar, et peut prendre part à l'action scénique, intervenir directement. Dans le théâtre conventionnel, on connaît les situations du spectateur discret et du spectateur invité (dans le théâtre de rue ou dans les performances des années 1960 par exemple). Mais le spectateur invisible est une situation inédite. On le connaît déambulant dans l'espace de représentation, mais on ne le connaît pas invisible, c'est-à-dire potentiellement absent : il peut très bien n'y avoir personne au bout du fil, la représentation peut avoir lieu sans public, et les acteurs n'en sauront rien si l'on décide de ne pas relever les statistiques de connexion sur le serveur.

Ainsi, dans les performances pour le réseau, parfois représentée, la salle est le plus souvent remplacée par un accès à la scène, cet accès définissant les modalités du point de vue et de la relation aux acteurs et aux autres spectateurs. La séparation entre la scène et la salle n'est plus une question de répartition dans l'espace mais de l'attribution d'une posture, et finalement de règles, de codes. Le spectateur est défini par des paramètres qui font partie du déroulement de la représentation. On détermine la marge de manœuvre, la circulation, jusqu'aux modalités de figurations du spectateur sur la scène. Et ce sont bien ces attributions qui permettent de différencier un acteur d'un spectateur lorsqu'ils sont représentés par des avatars similaires, davantage que sa position dans l'espace. Ce faisant, c'est dans la définition de ces paramètres que se joue la mise en scène des enjeux politiques du cyberespace, entre contrôle du réseau et utopies collectives. En effet, au-delà des sujets traités, le souhait de « faire du théâtre sur Internet » s'accompagne d'une réflexion sur l'accès à Internet considéré comme espace public, voire comme nouvelle agora, comme un espace vierge où peut s'exprimer la citoyenneté.

\title{
Localiser le spectateur mobile dans l'espace physique
}

\author{
«Les visions sont imprimées dans le téléphone » \\ Francis Picabia, Poème Banal, 1918
}

Plutôt que d'imaginer des modalités de présence des spectateurs sur le réseau, celui-ci est utilisé pour réunir des spectateurs à un même endroit. Ainsi des "flash mobs» ou rassemblements éclairs. Apparu en juin 2003 à New York, ce mouvement s'est depuis répandu dans les grandes métropoles du monde entier. Organisés par l'intermédiaire d'Internet, les flash mobs sont des rassemblements ponctuels, dans des espaces publics, de centaines de personnes qui ne se connaissent pas. A l'heure dite, elles exécutent une action rapide (dessiner sur le sol, jouer au jeu de l'oie, faire une ronde...) et se dispersent aussitôt après. Les instructions sont tenues secrètes jusqu'à la dernière minute et transmises par email 
et par SMS aux participants, qui doivent respecter à la lettre le scénario prévu par l'organisateur anonyme. Le réseau est l'objet de la manifestation. Ici plus d'acteurs ni de spectateurs, mais un rassemblement de personnes dans un même lieu physique, comme dans une perspective inversée du réseau, où le rassemblement n'est possible que dans des espaces virtuels et les corps absents physiquement. Les flash mobs, en occupant de manière fugitive et sans but des espaces publics, en les transformant pendant quelques minutes, sont une manifestation visible du flux des réseaux, voire des potentialités d'Internet et autres modes de communication mobiles comme outil d'organisation des communautés ${ }^{31}$.

Les technologies mobiles suscitent des téléscènes d'un nouveau genre, initiées sur et par le réseau mais se manifestant dans l'espace réel. D'une stratégie de délocalisation, de dispersion, on passe à une stratégie de localisation; du cyberespace au monde physique. Ce phénomène est lié aux nouveaux modes de présence des spectateurs. Équipés d'un attirail mobile et sans fil dont les formes nous sembleront très grossières d'ici peu, ils sont reliés en permanence à différents réseaux. Au théâtre, ce bric-à-brac électronique est malvenu car il perturbe la représentation. On demande donc aux spectateurs d'éteindre complètement toutes leurs extensions électroniques, pour que leur présence ne se manifeste pas pendant le déroulement du spectacle. Autrement dit, le nouveau statut du spectateur, son corps-interface, n'est pas encore pris en compte dans les représentations qui conservent le face à face entre acteurs et spectateurs. On le renvoie à son ancien statut, alors que les signes, ou plutôt les signaux, de sa présence, se sont transformés.

Aujourd'hui, les dispositifs de présence à distance s'intéressent de prêt à ce corps signal, ce corps récepteur et réceptif, mettant au centre du débat la mobilité, dans une pratique renouvelée du nomadisme. L'émergence de nombreux festivals autour de la notion de mobilité semble prendre la mesure de ce phénomène. De plus en plus d'artistes réalisent des œuvres pour nos extensions mobiles et numériques. L'une des œuvres pionnières dans ce domaine est le concert de sonneries de téléphones portables, Dialtones, organisé par Golan Levin lors du festival Ars Electronica en 2001. Sur le même mode, Antoine Schmitt et JeanJacques Birgé ont réuni 100 lapins Nabaztag apportés par leurs propriétaires afin d'interpréter un opéra ${ }^{32}$ spécialement composé pour ces rongeurs numériques, les sons et les mouvements (lumières et chorégraphie d'oreilles) étant transmis par wifi à chacun des lapins.

Mais c'est le téléphone portable qui reçoit la faveur des artistes, après quelques expériences menées avec des walkmans ou des audio guides ${ }^{33}$. Le parcours du metteur en scène Stefan Kaegi suit cette évolution technologique. En 1999, avec son comparse Bernd Ernst, il crée Kanal Kirchner, œuvre présentée au festival Spielart à Munich. Le spectateur, muni d'un walkman, part dans les rues de Munich en suivant les instructions données par une

${ }^{31}$ Ce point est développé par Howard Rheingold in Smart Mobs : The Next Social Revolution, Cambridge, MA, Perseus Publishing, 2002.

${ }^{32}$ Nabaz'mob, opéra pour 100 lapins communicants, Antoine Schmitt et Jean-Jacques Birgé, Centre Pompidou, 2006.

${ }^{33}$ Sur cet aspect, voir Balme C. B., " Audio theatre : the mediatization of theatrical space », in Intermiediality in Theatre and Performance, Rodopi B.V., 2006, p. 117-124. 
voix enregistrée, sur les traces d'un libraire disparu de manière étrange. À la réalité de la ville, une fiction se superpose, dédoublant la perception du spectateur entre ville réelle et ville imaginaire. Le spectateur devient alors un acteur de la ville imaginaire, engagé dans un thriller dont il est partie prenante, tout en évoluant dans les rues de Munich, mû par une voix invisible. Avec l'engouement pour l'Ipod et autres baladeurs numériques, combiné au développement du podcast, il ne serait pas étonnant de voir ces nouvelles formes de radiothéâtre se répandre.

Quelques années plus tard, Stefan Kaegi renouvelle l'expérience, cette fois au sein de la compagnie Rimini Protokoll, et passe du walkman au téléphone portable. Selon la compagnie, il s'agirait de la toute première représentation théâtrale pour téléphone portable («mobile phone theatre »). Nommé Call Cutta, ce spectacle a eu lieu à Calcutta du 26 février au 30 avril 2005. Les concepteurs partent de la réalité des centres d'appels téléphoniques en Inde à destination de clients américains et anglais. Dans les dialogues qui s'engagent entre clients occidentaux et opérateurs, tout est fait pour éluder l'origine indienne des seconds, en commençant par nier ces signes distinctifs que sont les prénoms et les accents locaux, et en finissant par faire croire à leurs interlocuteurs qu'ils connaissent comme leur poche ce petit coin de terre dans le fin fond du Mississipi.

Call Cutta est basé sur le même principe de redoublement/dédoublement de la ville que Kanal Kirchner. Le spectateur suit les instructions qui lui sont données par un ou des acteurs situés dans un «call centre ». Au travers d'une conversation qui va révéler la personnalité réelle des acteurs, dans un glissement du faux-américain au vrai-indien, le spectateur est guidé dans la ville, en fonction de différentes trames dramaturgiques : un récit d'espionnage, une pièce historique sur l'indépendance du Bengale... Parfois, l'appel est interrompu, le spectateur invité à prendre un café, il sera rappelé plus tard. Il est alors propulsé, le temps d'une pause, dans la ville réelle. Dans une seconde phase du projet, l'acteur est toujours situé dans un centre d'appel à Calcutta, tandis que le spectateur est à Berlin. Ils échangent des informations sur la ville, partagent des confidences, le spectateur entre dans des lieux inconnus et mystérieux, guidé par la voix d'une personne qui n'est jamais allée à Berlin.

Toujours en 2005, mais de l'autre coté de l'Atlantique, Robert Whitman, artiste s'intéressant depuis les années 1960 à l'intégration des technologies dans les représentations théâtrales, crée une performance pour des téléphones portables intitulée Local report. Celleci a eu lieu à cinq reprises, dans cinq centre commerciaux américains différents. À chaque fois, Whitman confie à trente personnes volontaires des téléphones portables dernier cri munis de caméras vidéo, avec la consigne de filmer leur environnement quotidien en le commentant pendant une demi-heure. Whitman monte les différentes sources audio et vidéo en direct. Le résultat est diffusé sur un écran dans le centre commercial et sur Internet. Cette performance est l'actualisation avec des procédés nouveaux d'une œuvre créée en 1972, News, dont la première a eu lieu à New York : les personnes étaient éparpillées dans la ville et devaient appeler Whitman depuis une cabine téléphonique toutes les 5 minutes, pour lui 
décrire ce qu'ils voyaient. Whitman retransmettait en direct ces interventions sur une chaîne de radio, les coupant chaque fois que «la personne qui appelait produisait une image cohérente $^{34} »$, refusant toute description reconnaissable, identifiable, réaliste du lieu évoqué.

Dans ce type de théâtre, le spectateur ne peut être invisible : la représentation s'invite directement chez lui, dans ses extensions électroniques, dans son intimité. La perspective est renversée : il ne s'agit pas d'inviter le spectateur à se connecter sur le réseau, à se brancher sur une scène virtuelle, mais de rejoindre le spectateur là où il se trouve, et de faire du lieu où il est, de la ville dans laquelle il évolue, la scène potentielle, la scène virtuelle, du drame dont il va être partie prenante.

C'est ce que souhaite explorer un nouveau festival, First Play Berlin, dont la première a eu lieu en novembre 2006. Il s'agit d'un « programme international d'art numérique vivant fusion de la performance et de la technologie mobile. Étendant les limites de l'interaction et de la participation, le regardeur est invité à naviguer dans des espaces virtuels en effectuant un voyage dans le monde réel, physique ». Entre le jeu vidéo, l'art radiophonique et le théâtre, ces œuvres proposent de guider le spectateur dans des univers virtuels qui s'appuient sur l'espace réel, en le munissant de téléphones portables ou de PDA. Ainsi, dans Our House, Daniel Belasco Rogers propose au spectateur de découvrir la maison de son enfance et sa famille. Dans un local, au mur, des photographies jaunies collées. Lorsque le spectateur s'approche d'une photo, sa présence est détectée. La famille de l'artiste lui confie alors souvenirs et commentaires à l'oreille (leur voix enregistrée est diffusée dans un casque), tandis que des images s'affichent sur le PDA qui lui a été remis. Dans Life: a user's manual, Michelle Téran propose à un groupe de personnes une excursion dans la ville. Munie d'un système qui lui permet de capter les images filmées par les caméras de surveillances privées, elle révèle aux spectateurs des scènes de surveillance ordinaire. Autre oeuvre, qui utilise à nouveau le téléphone portable : Day of the Figurines, par Blast Theory, développée dans le cadre du programme « City as Theatre » du projet européen IperG. Tous les participants sont convoqués sur le lieu du festival. Ils choisissent une figurine, la baptisent, définissent sa personnalité. Puis ils la situent à un endroit précis d'une ville imaginaire. Au fur et à mesure des 24 journées suivantes, une journée représentant une heure dans le monde fictif, il va recevoir et émettre des SMS qui vont lui permettre de guider son personnage dans cet univers et entrer en contact avec d'autres joueurs. L'objet des recherches d'IperG est la création de "pervasive games», dont la traduction littérale est jeux envahissants, et dont la caractéristique majeure est d'avoir lieu dans le cadre de notre vie quotidienne, provoquant une contamination entre le jeu et la vie. Autrement dit, il s'agit de créer de nouvelles manières de jouer en utilisant comme interface l'objet quotidien sans fil le plus répandu, à savoir le téléphone portable. Day of the Figurines est une première tentative dans ce sens, articulée autour d'une dramaturgie précise qui prend en compte la présence du spectateur et son engagement dans la représentation.

\footnotetext{
${ }^{34}$ Cité in Local Report, [http://www.whitmanlocalreport.net/sub_project.htm].
} 
Le titre de l'œuvre de Robert Whitman, Local Report, est en soit tout un programme : il s'agit bien de s'ancrer dans un lieu existant, d'en révéler la nature particulière, spécifique, en allant chercher le spectateur là où il se trouve. Et aujourd'hui, là où il se trouve, c'est à côté de son téléphone portable (qui est aussi une caméra), en attendant qu'on le lui greffe sous la peau. Cette préoccupation pour l'espace physique dans lequel évolue le spectateur est au cœur d'un mouvement dénommé «locative media », né au cours d'un workshop organisé à Litvia en $2003^{35}$. Les «médias locatifs» (au sens des prépositions locatives) sont nés de la combinaison des technologies mobiles et des systèmes d'information géographique (GIS : Geographic Information Systems) comme le GPS, le wifi, ou encore les RFID. Cette combinaison permet de reconsidérer la manière dont on perçoit et l'on investit l'espace. $\mathrm{Ou}$ encore : comment habiter l'espace contemporain à l'heure de Google Earth et du téléphone mobile, lequel est aussi un outil de surveillance de ses utilisateurs ${ }^{36}$. De nombreuses recherches, regroupées sous les termes de Ubiquitous computing ou Ambient Intelligence, concernent ces aspects, cherchant à développer un accès différent aux informations numériques en les rendant dépendantes de leur contexte de consultation. Le renversement de perspective est d'importance : il ne s'agit plus de créer un monde virtuel ex-nihilo, mais de construire un espace de données à partir d'un espace réel, et de jouer de leurs coïncidences ou de leurs dissonances. Cette stratégie opère la " désacralisation pratique de l'espace ${ }^{37}$ » qui pour Michel Foucault n'était pas encore effective. En effet, elle rompt les « oppositions que nous admettons comme toutes données : par exemple, entre l'espace privé et l'espace public, entre l'espace de la famille et l'espace social, entre l'espace culturel et l'espace utile, entre l'espace de loisirs et l'espace de travail ${ }^{38} \gg$.

Délocaliser/localiser le spectateur : quel que soit le mode opératoire, espace physique et espace numérique, loin de s'opposer, s'hybrident et se contaminent l'un l'autre. Leur continuité rapproche le spectateur de la scène, les lie l'un à l'autre dans une relation qui favorise un aller-retour constant entre immersion et mise à distance. Si bien que la coupure sémiotique entre la scène et la salle disparaît au profit d'une couture entre espace physique et espace numérique, le rapport entre ces deux derniers n'étant pas un rapport scène-salle.

Ce faisant, la présence à distance, alliée à une médiation à double sens (au contraire du sens unique de la radio - de l'émetteur vers le récepteur), remet en cause l'opposition entre le spectateur et l'acteur, entre le voir et l'agir - ce que certains ont appelé le « spectacteur ${ }^{39}$ ».

\footnotetext{
${ }^{35}$ Voir le dossier coordonné par Drew Hemment, « Locative Media », Leonardo Electronic Almanac, Vol. 14, $\mathrm{n}^{\circ}$ 03-04, juillet 2006, [http://leoalmanac.org].

${ }^{36}$ On sait comment ce type de données a pu être utilisé par l'armée russe pour localiser et éliminer des chefs tchétchènes. Aujourd'hui, le laboratoire Senseable Lab au MIT développe des projets d'urbanisme et de gestion du territoire en fonction des informations fournies par les téléphones portables des citadins.

${ }^{37}$ Foucault M., « Des espaces autres », conférence au Cercle d'études architecturales, 14 mars 1967, in Dits et écrits, T. IV, NRF, 1994, pp. 752-762.

${ }^{38} \mathrm{Idem}$.

${ }^{39}$ Weissberg J.L., Présences à distance. Déplacement virtuel et réseaux numériques : pourquoi nous ne croyons plus à la télévision, L'Harmattan, Paris, 1999 (publié sur Internet: [http://hypermedia.univparis8.fr/Weissberg/presence/presence.htm]; voir également: Weissberg J.-L., Amato E. A., "Le Corps à l'épreuve de l'interactivité : interface, narrativité, gestualité », in Anomalie Digital_arts "Interfaces », n $\mathrm{n}^{\circ}$,
} 
Faire et voir ne sont plus opposés, favorisant ainsi l'émergence de nouvelles postures de l'acteur et du spectateur. L'un et l'autre peuvent êtres considérés comme des « joueurs » (au sens de jouer une pièce) et il ne s'agirait pas tant de distinguer le voir du faire, que le geste amateur (le spectateur) du geste expert (l'acteur). Tout comme le faire et le voir, l'actif et le passif, le physique et le virtuel, la scène et la salle, ne peuvent être strictement opposés et renvoyés dos à dos dans une logique binaire, tout comme ces couples se combinent et se contaminent à l'infini, il n'est plus possible d'opposer la présence à l'absence dans les téléscènes ${ }^{40}$. Selon les interfaces, cette présence se déploie dans une gamme composée de différents degrés. Le face à face physique entre l'acteur et le spectateur peut alors être considéré comme l'un des degrés de cette gamme. Le problème n'est alors pas tant que les spectateurs soient présents aux acteurs, et vice-versa, que d'établir les modalités de la présence du joueur dans le dispositif.

mars 2003, p. 41-51. Le terme « spect-acteur » a d'abord été employé par Augusto Boal dans les années 1960 pour désigner le spectateur qui monte sur scène dans le Théâtre de l'opprimé. Cependant, Jean-Louis Weissberg ne fait pas référence à Boal lorsqu'il emploie ce terme.

${ }^{40}$ Katherine N. Hayles développe une analyse semblable pour les textes littéraires et les corps humains. Elle propose de remplacer le couple présence/absence par celui de structure/aléatoire. Voir Hayles K. N., How we became Posthuman : Virtual Bodies in Cybernectics, Literature and Informatics, University of Chicago Press, 1999. 